a familia Solanaceae comprende cerca de 90 géneros y alrededor de 2,600 especies de distribución subcosmopolita. La familia es especialmente diversa en Sudamérica, en donde se han registrado 56 géneros, de los cuales 25 son endémicos de esa región (Mabberley, 1990). El género Juanulloa Ruiz et Pav. es exclusivo de América y contiene alrededor de diez especies tropicales, predominantemente epífitas. Nee (1986) señala que la mayoría de las especies de este género son sudamericanas, con algunos taxa en Centroamérica y México.

Durante exploraciones botánicas recientes se encontró un individuo de una solanácea epífita en el municipio de Mascota, Jalisco. Al identificarlo, coincidió con la descripción de Juanulloa mexicana. Con la revisión posterior en herbarios, se localizaron un par de especímenes adicionales provenientes del municipio de Cabo Corrientes, Jalisco, las cuales confirmaron el hallazgo. Se analizó la distribución geográfica de la especie y se concluyó que las colectas presentes representan registros nuevos para el occidente de México, lo cual significa una ampliación significativa hacia el oeste de su distribución geográfica conocida. El objetivo del presente trabajo es documentar la presencia de Juanulloa mexicana en el occidente de México.

Se realizaron dos viajes exploratorios en el municipio de Mascota, Jalisco, en 2001 y 2004. El material colectado de Juanulloa mexicana se identificó con base en material cultivado y en especímenes herborizados depositados en el herbario (MEXU) de la Universidad Nacional Autónoma de México y en los herbarios IBUG, IEB, MEXU y XAL. En MEXU se revisó material adicional proveniente de Jalisco, Veracruz, Oaxaca, Chiapas y Tabasco.

Los individuos de J. mexicana en

\title{
JuANUlloa meXicana (SOlanaceae), NUEVO REGISTRO EN EL OCCIDENTE DE MÉXICO
}

\author{
Yalma Luisa VarGas-RodrigueZ ${ }^{1,4}$, Miguel de Jesús \\ ChÁZARO-BASÁÑEZ ${ }^{2}$ \\ Y JosÉ ANTONIO VÁZQUEZ-GARCíA ${ }^{3}$
}

'Louisiana State University, Department of Biological Sciences, 107 Life Sciences Building, Baton Rouge 70803, Louisiana, Estados Unidos de América.

${ }^{2}$ Universidad de Guadalajara, Centro Universitario de Ciencias Sociales y Humanidades, Departamento de Geografía y Ordenación Territorial, Av. de Los Maestros y Mariano Bárcenas, Guadalajara 44260, Jalisco, México.

${ }^{3}$ Centro Universitario de Ciencias Biológicas y Agropecuarias, Departamento de Botánica y Zoología, km 15 carretera Guadalajara-Nogales, Las Agujas, Zapopan 45110, Jalisco, México.

${ }^{4}$ Autor para la correspondencia. Correo-e: yvarga1@|su.edu

Jalisco presentan poca variación morfológica y se considera que no difieren significativamente del resto de los ejemplares examinados para el país. En México, la especie se registra en Chiapas (Standley, 1924; Breedlove, 1986; Acevedo, 1988), Tabasco [J. Calónico 21323 (MEXU), S. Zamudio 1475 (MEXU), Guadarrama s. n. (MEXU)], Veracruz (Standley, 1924; Nee, 1986), Oaxaca (Standley, 1924). En Guerrero sólo fue recolectada en 1937 en el municipio de La Unión de Isidoro Montes de Oca [Hinton et al. s. $n$. (K)]. En Durango se encontró en 1906, en el municipio de Canatlán [E. Palmer 169 (MO)]; sin embargo, la especie no fue incluida en el listado florístico del estado (GonzálezElizondo et al., 1991). Vargas y Rodríguez (2001) citan la presencia de 19 géneros y 150 taxa infragenéricos de Solanaceae para Jalisco, pero no incluyeron a J. mexicana, a pesar de que existen dos ejemplares depositados en herbarios [O. Téllez 5886 (MEXU), G. Castillo-Campos, P. Zamora y R. Acevedo 10464 (XAL)], ambos de Cabo Corrientes y recolectados en 1982 y 1993, respectivamente. Esto sugiere que Vargas y Rodríguez (2001) sólo consultaron principalmente herbarios jaliscienses tales como IBUG, GUADA y ZEA, omitiendo herbarios con mayor representatividad de la flora mexicana, tales como MEXU y ENCB. Caso semejante se presenta en el trabajo de Cuevas-Arias (2000) en su tesis sobre diversidad de la familia Solanaceae en Jalisco.

Juanulloa mexicana se distribuye desde México (Durango, Jalisco, Guerrero, Veracruz, Oaxaca, Chiapas y Tabasco) hasta Centroamérica, Colombia, Ecuador y Perú (figura 1). En Jalisco la especie presenta una distribución muy restringida. Sólo se han encontrado dos individuos en 


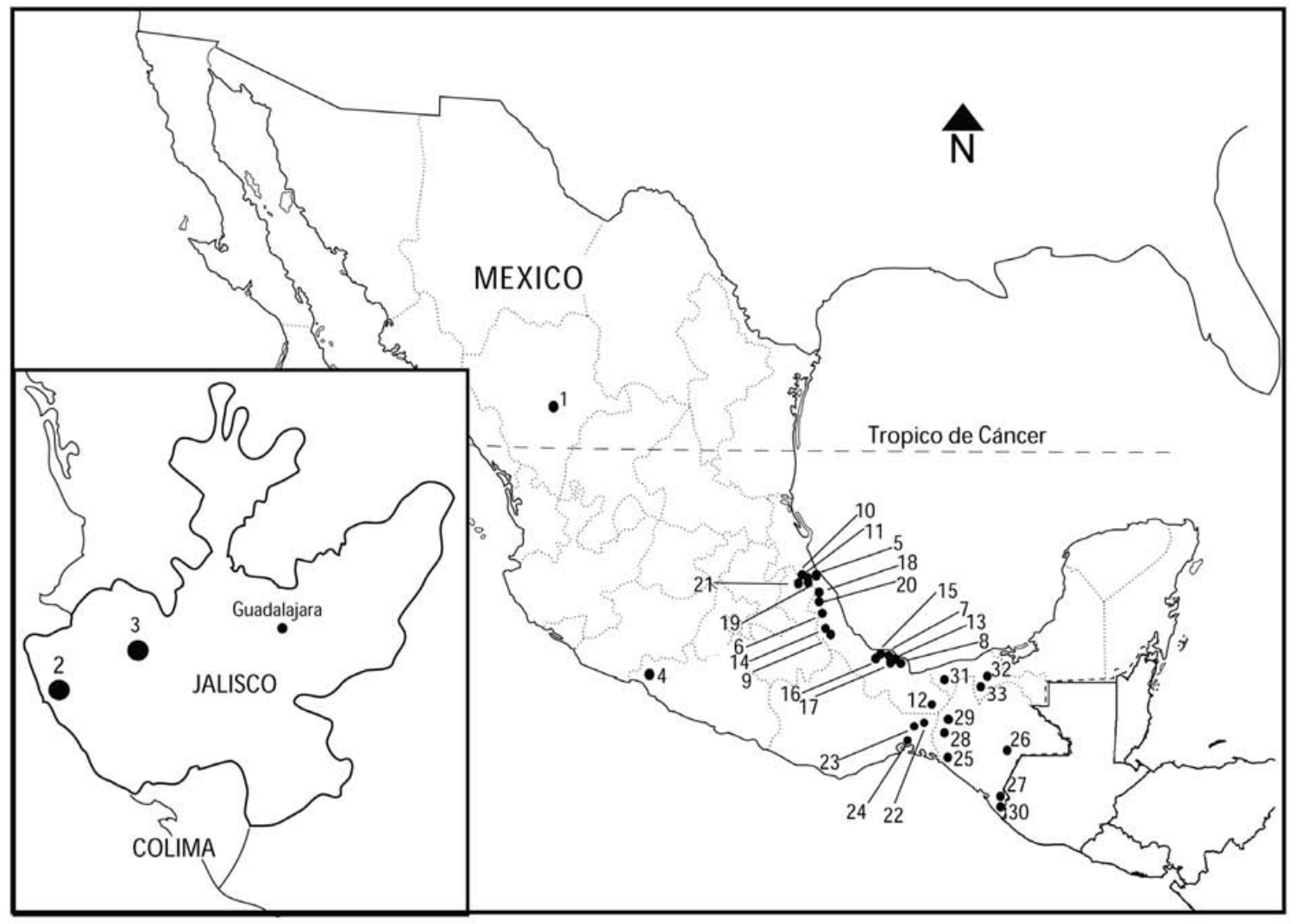

Figura 1. Distribución conocida de Juanulloa mexicana en México. Se muestran los municipios en los que se registró su presencia en México. DURANGO: 1. Canatlán [E. Palmer 169 (MO)]. JALISCO: 2. El Tuito, Cabo Corrientes [O. Téllez 5866 (MEXU)], 3. Barranca Coamil del Naranjo, Mascota [Y. L. Vargas-Rodriguez, M. Cházaro-B. y A. Vázquez-García 435 (IBUG)]. GUERRERO: 4. La Unión de Isidoro Montes de Oca [Hinton et al. s.n. (K)]. VERACRUZ: 5. Actopan [G. Webster y M. Breckon 15426 (XAL)], 6. Atoyac [E. Matuda 1396 (XAL)], 7. Catemaco [A. Gómez-Pompa 4524 (XAL)], 8. Coatzacoalcos [Ll. Williams 8801 (XAL)], 9. Cosamaloapan de Carpio [G. Martínez 2233 (XAL)], 10. Emiliano Zapata [Calzada 10173 (MEXU)], 11. Jalcomulco [G. Castillo-Campos et al. 14178 (XAL)], 12. Jesús Carranza [M. Vázquez, I. Navarrete y A. García 2527 (XAL)], 13. Mecayapan [G. Castillo-Campos et al. 13873 (XAL)], 14. Omealca $[R$. Robles 276 (XAL)], 15. San Andrés Tuxtla [D. Lorente 3466 (XAL)], 16. Santiago Tuxtla [F. Ventura 14016 (XAL)], 17. Soteapa [J. Calzada 11374 (XAL)], 18. Tenampa [C. Purpus 2435 (XAL)], 19. Totutla [Ventura 3654 (ENCB; TEX)], 20. Xalapa [G. Carmona 53 (XAL)], 21. Xico [I. Acosta 203 (XAL)]. OAXACA: 22. Santa María Chimalapa [S. Maya 4361 (MEXU)], 23. Asunción Ixtaltepec [R. Torres y R. Cedillo 105 (XAL)], 24. Santo Domingo Tehuantepec [M. Torres, R. Torres y C. Martínez 28 (XAL)]. CHIAPAS: 25. Angel Albino Corzo [Heath y Long 928 (MEXU)], 26. Tzimol [Reyes G. 492 (MEXU)], 27. Cacahoatan [E. Ventura y E. López 276 (XAL)], 28. Ocozocoautla de Espinoza [J.I. Calzada 9807 (XAL)], 29. Tecpatan [F. Vázquez. 1080 (XAL)], 30. Tuxtla Chico [E. Martínez y A. Márquez, 19898 (XAL)]. TABASCO: 31. Huimanguillo [J. Calónico 21323 (MEXU)], 32. Macuspana [S. Zamudio 1475 (MEXU)], 33. Teapa [Guadarrama s.n. (MEXU)].

una localidad cercana a El Tuito, municipio de Cabo Corrientes, y otro en la barranca del Coamil del Naranjo, municipio de Mascota, ambos en la vertiente costera de Jalisco (figura 1).

En cuanto a su abundancia, en el occidente de México se considera rara, encontrándose individuos solitarios. En un inventario florístico del municipio de Cabo Corrientes, Jalisco (Hernández del Toro, 2003), no se encontró la especie. La sierra de Manantlán y la bahía de Chamela son las áreas más conocidas y exploradas florísticamente en Jalisco; no obstante, la especie no ha sido encontrada en ellas (Lott, 1985, 1993; Vázquez-García et al., 1995). La colectas de Durango y Guerrero datan de hace 100 y 69 años, respectivamente, a pesar de haberse incrementado la intensidad de la exploración botánica en ambos estados en los últimos años, aunque el hábito epífito de la especie podría hacerla inconspicua para los colectores. Sólo en el oriente de México (Veracruz) las colectas han sido numerosas, pero restringidas a unos cuantos sitios como la 
Estación de Biología Tropical Los Tuxtlas. En Jalisco, la tala de encinares y la acelerada transformación del bosque tropical caducifolio en matorrales secundarios constituyen la principal amenaza para la población de J. mexicana, debido a su hábito epífito.

En las poblaciones de Jalisco, se observó la floración en febrero, marzo y abril y fructificación en junio. Sin embargo, Nee (1986) señala que la floración ocurre durante todo el año. Las flores son polinizadas por colibríes (Nee, 1986). Se han observado larvas de las mariposas Melinaea ethra y M. scylax como herbívoros de J. mexicana.

La especie habita en bosques tropicales subcaducifolios o perennifolios (selva baja y selva alta perennifolia) y en bosques de encino, generalmente cerca de ríos o arroyos. En Jalisco crece en bosque de encino y en su ecotono con el bosque tropical caducifolio. En Costa Rica se le ha observado sobre Inga sp. y Terminalia sp. (INBio, 1998); en una localidad de Guerrero y Veracruz crece sobre Quercus sp., en tanto que en Jalisco crece sobre Lysiloma sp. Se desarrolla desde el nivel del mar hasta $1,300 \mathrm{~m}$ de altitud.

\section{EJEMPLARES EXAMINADOS: MÉXICO: Jalisco, $6 \mathrm{~km}$ al O de El} Tuito, camino Chacala-Cabo Corrientes, bosque de encino, 30 junio 1982, O. Téllez 5866 (MEXU); La Puerta, municipio de Cabo Corrientes, 500 m s.n.m., 20²6' N, $105^{\circ} 18^{\prime}$ O, bosque de Quercus, FL, 27 febrero 1993, G. CastilloCampos, P. Zamora y R. Acevedo 10464 (XAL); barranca del Coamil del Naranjo, municipio de Mascota,
1,100 m s.n.m., arbusto epífito, estéril (hojas), 7 octubre 2001, $M$. Cházaro-B., I. Contreras V. y $J$. Cortés A. 8142 (IBUG); creciendo a lo largo del río Mascota, ecotono de bosque de encino y bosque tropical caducifolio, FL, 25 abril 2004, Y. L. Vargas-Rodriguez, M. Cházaro-B. y A. Vázquez-García 435 (IBUG); estéril (hojas) y en cultivo, 28 junio 2004, A. Vázquez-García 7829 (IBUG).

\section{Agradecimientos}

Agradecemos a Oscar M. Valencia, Ignacio Contreras V., Jesús Cortés A., Apolinar Gómez N., Rafael Castillón H. y Ramón Briseño por su asistencia en el trabajo de campo. José A. Lomelí Sención, Raúl Acevedo, Nelly Diego y un revisor anónimo hicieron importantes sugerencias para mejorar este trabajo. M. Cházaro-B. agradece al Departamento de Geografía del CUCSH de la Universidad de Guadalajara por el apoyo académico y financiero a sus proyectos de investigación.

\section{Literatura citada}

Acevedo R. 1988. La vegetación de la Sierra de Atoyac. Tesis de Licenciatura, Facultad de Ciencias Biológicas, Universidad Veracruzana, Xalapa, 79 pp.

Breedlove D.E. 1986. Listados Florísticos de México IV. Flora de Chiapas. Instituto de Biología, Universidad Nacional Autónoma de México, México, D.F.

Cuevas-Arias C.T. 2000. Distribución y riqueza de la familia Solanaceae en Jalisco. Tesis de Licenciatura, Centro Universitario de Ciencias Biológicas y Agropecuarias, Universidad de Guadalajara, Zapopan, 109 pp.
González-Elizondo M., GonzálezElizondo S. y Herrera Arrieta Y. 1991. Listados Florísticos de México IX. Flora de Durango. Instituto de Biología, Universidad Nacional Autónoma de México. México, D.F.

Hernández del Toro I.M. 2003. Flora y vegetación de entre los ríos Tecolotlán y María García, municipios de Cabo Corrientes y Tomatlán, Jalisco, México. Tesis doctoral, Universidad de Salamanca, Salamanca, 521 pp.

INBio [Instituto Nacional de Biodiversidad]. 1998. Manual de Plantas de Costa Rica. Instituto Nacional de Biodiversidad, Santo Domingo de Heredia, Costa Rica.

Lott E.J. 1985. Listados Florísticos de México III. La Estación de Biología de Chamela, Jalisco. Instituto de Biología, Universidad Nacional Autónoma de México, México, D.F.

Lott E.J. 1993. Annotated checklist of the vascular flora of the Chamela Bay region, Jalisco, México. Occasional Papers of the California Academy of Sciences 148:1-60.

Mabberley D.J. 1990. The Plant Book. Cambridge University Press, Cambridge.

Nee M. 1986. Solanaceae I. Flora de Veracruz. Fascículo 49. Instituto Nacional de Investigaciones sobre Recursos Bióticos, Xalapa.

Standley P.C. 1924. Trees and shrubs of Mexico. Contributions from the United States National Herbarium 23:1278.

Vargas O. y Rodríguez A. 2001. Diversidad de la familia Solanaceae en el estado de Jalisco. ScientiaCUCBA 3:85-91.

Vázquez-García J., Cuevas G.R., Cochrane T.S., Iltis H.H., Santana M.F. y Guzmán H.L. 1995. Flora de Manantlán. Universidad de Guadalajara-IMECBIO, University of Wisconsin Madison, Botanical Research Institute of Texas, Fort Worth. 